\title{
Physical Characterization and Elaboration Discussion of a Clay-PEG 6000 Composite with Natural Clay Matrix
}

\author{
Iatimad Akhrif ${ }^{*}$, Mostapha El Jai ${ }^{2}$, Laila Mesrar ${ }^{1}$, Ahmed Elkhalfi ${ }^{3}$, Abdelhamid Touache ${ }^{3}$, \\ Raouf Jabrane ${ }^{1}$ \\ ${ }^{1}$ Geosciences and Environment Laboratory, Faculty of Sciences and Techniques, Sidi Mohammed Ben Abdellah \\ University, Fez, Morocco \\ ${ }^{2}$ Advanced Material and Application Team, Ecole Nationale Supérieure d'Arts et Métiers de Meknès, Moulay \\ Ismail University, Meknes, Morocco \\ ${ }^{3}$ Mechanical Engineering Department, Faculty of Sciences and Techniques, Sidi Mohammed Ben Abdellah \\ University, Fez, Morocco \\ Email: ${ }^{*}$ akhrif.iatimad@gmail.com, mostaphaeljai@gmail.com, lailamesrar@yahoo.fr, aelkhalfi@gmail.com, \\ atouache@yahoo.fr, raouf.jabrane@usmba.ac.ma
}

Received 6 April 2014; revised 6 May 2014; accepted 13 May 2014

Copyright (C) 2014 by authors and Scientific Research Publishing Inc.

This work is licensed under the Creative Commons Attribution International License (CC BY). http://creativecommons.org/licenses/by/4.0/

(c) (i) Open Access

\begin{abstract}
In this paper, we compare different nanoclay-PEG composites and the influence of the input parameters especially the percentage of PEG and the clay size. Because of the facility of material elaboration, dried state with grinding, we adopted a complete experiments plan to obtain a maximum of robustness of the responses. For each sample, we made an XRD analysis to see if we obtain the intercalation of the PEG 6000 (Polyethylene Glycol 6000) within the clay sheets. The characterization adopted consists on the measurement of the shrinking of some cylinders we made, the liquidity and plasticity limits according to the Casagrande protocol used in geotechnical clays characterizations. We utilize also the methylen blue protocol to estimate the variation of the specific surface of ionic exchange of the clay sheets according to the PEG 6000 percentage and the clay sizes. SEM microscopy permits to visualize some of the phases detected by the XRD analysis. The TEM microscopy permits also to see the amorphous phases created by the grinding protocol which affects significantly the specific surface and the shrinking of the new materials. For each section, we made some conclusions with interpretation in order to integrate these results in civil engineering, classical/artisanal material construction and geotechnical fields.
\end{abstract}

\section{Keywords}

Clay Structure, PEG 6000/Clay Matrix Composite, ICP Analysis, Atterberg Limits, Specific Surface,

*Corresponding author.

How to cite this paper: Akhrif, I., El Jai, M., Mesrar, L., Elkhalfi, A., Touache, A. and Jabrane, R. (2014) Physical Characterization and Elaboration Discussion of a Clay-PEG 6000 Composite with Natural Clay Matrix. Engineering, 6, 338-354. 


\section{Introduction}

The exploration of nanoclay doping has known, in the last decades, a very large interest because of the deeper effect that nanometer modifications can give to the corresponding matrix of clay, compared to macroscopic modification. Wide researches were made on several kinds of reinforcing materials, of which the most important such as metallic/metallic oxides particles [1] and polymers for increasing some of the mechanical and physicochemical properties of the material. But the difficulty is the rarefy of the studies corresponding to natural material, compared to the number of subjects that treat the modification of industrial and purified clay structure [2]-[5].

We are interested here to the development of new biomaterials dedicated to construction industry, which could be built by natural clays and simple methods, and where every person in our region could elaborate itself. The material should have compromise between its mechanical, physico-chemical and thermal properties, such as elasticity, mechanical resistance, erosion resistance, conduction and thermal capacity, etc.

In this study, we are working on the consolidation of the clays of Fez region in Morocco by adding the PEG 6000. In a future works, we will study the difference between samples taken from different points (horizontal variation).

The mixing method consists on a simple grinding of the clay and PEG 6000 according to three different clay sizes scales. The PEG 6000 percentage varies from $0.1 \mathrm{~g}$ per $6 \mathrm{~g}$ of clay to $0.6 \mathrm{~g}$ PEG per $6 \mathrm{~g}$ of clay.

So the analysis consists to evaluate the influence of each input parameters on the physical properties studies: liquidity (Casagrande protocol) and plasticity limits, the shrinking depending on the time (10 days were adopted measured on diameters, heights and mass of multiple little laboratory cylinders) and specific surface of the clay estimated by the methylen blue protocol.

The resulting measurements were plotted to visualize the effect of the input parameters on the physical properties.

Regarding the geotechnical properties, liquidity and plasticity limits, specific surface... they permit to define the material state on the stage of elaboration. Hence, this characterization is very important to optimize the time of processing and the row materials used for this purpose.

\section{Experimentation Plan and the Corresponding Input and Output}

\subsection{Input Parameters and Experiments Plan Design}

The complete plan corresponds to 3 clay sizes and 6 PEG percentages. It corresponds to 18 essays.

Table 1 gives an overview of the values of the inputs adopted with the correspondent designation.

The masses of PEG 6000 given in the Table 1 are mixed with $6 \mathrm{~g}$ of natural clay.

The $0 \%$ (0 g) PEG 6000 corresponds to natural clay with no modification. It permits us to have a global comparison with the modified samples. The essays with 0\% PEG 6000 are denoted 19, 20 and 21.

The detail of the plan we followed is given according to Table 2.

The processing of the material corresponds to a simple grinding of the clay and PEG 6000 according to the experiments plan of Table 2. The grinding/mixing takes $15 \mathrm{mn}$ on a RETSCH RM 200 Mortar grinder machine, presented on Figure 1.

The clay size can be adjusted on three different sizes by acting on the pressure handle, shown on Figure 1 .

\subsection{Output: Physical Properties Measured and the Correspondent Protocols}

The output corresponds to the measurement of some of the principal physical properties of the materials. Each

Table 1. Plan factors and corresponding levels.

\begin{tabular}{|c|c|c|c|c|c|c|c|}
\hline \multirow{2}{*}{$\begin{array}{c}\text { Factor } \\
\text { Clay size }\end{array}$} & \multicolumn{7}{|c|}{ Levels } \\
\hline & \multicolumn{3}{|c|}{1 (size 3) } & \multicolumn{2}{|c|}{2 (size 5) } & \multicolumn{2}{|c|}{3 (size 7) } \\
\hline PEG\% & $\mathbf{0 g}$ & 1 (0.1 g) & 2 (0.2 g) & 3 (0.3 g) & $4(0.4 \mathrm{~g})$ & $5(0.5 \mathrm{~g})$ & $6(0.6 \mathrm{~g})$ \\
\hline
\end{tabular}




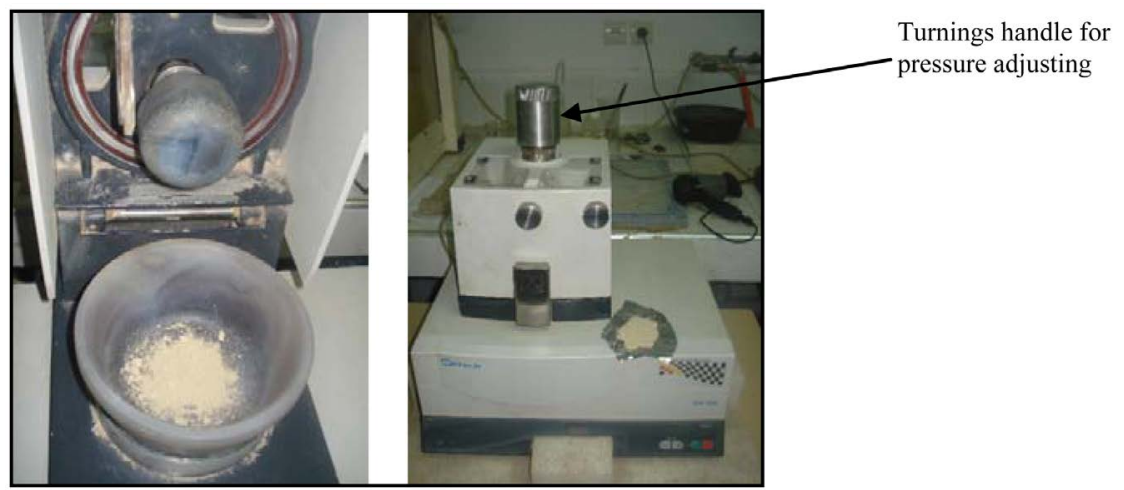

Figure 1. RETSCH RM 200 mortar grinder.

Table 2. The detail of the experiments-complete plan.

\begin{tabular}{cccccc}
\hline No. experiment & PEG level & Size level & No. experiment & PEG level & Size level \\
\hline $\mathbf{1}$ & 1 & 1 & $\mathbf{1 0}$ & 4 & 1 \\
$\mathbf{2}$ & 1 & 2 & $\mathbf{1 1}$ & 4 & 2 \\
$\mathbf{3}$ & 1 & 3 & $\mathbf{1 2}$ & 4 & 3 \\
$\mathbf{4}$ & 2 & 1 & $\mathbf{1 3}$ & 5 & 1 \\
$\mathbf{5}$ & 2 & 2 & $\mathbf{1 4}$ & 5 & 2 \\
$\mathbf{6}$ & 2 & 3 & $\mathbf{1 5}$ & 5 & 3 \\
$\mathbf{7}$ & 3 & 1 & $\mathbf{1 6}$ & 6 & 1 \\
$\mathbf{8}$ & 3 & 2 & $\mathbf{1 7}$ & 6 & 2 \\
$\mathbf{9}$ & 3 & 3 & $\mathbf{1 8}$ & 6 & 3 \\
$\mathbf{1 9}$ & 0 & 1 & $\mathbf{2 1}$ & 0 & 3 \\
$\mathbf{2 0}$ & 0 & 2 & & & \\
\hline
\end{tabular}

characteristic is calculated/measured by a standard protocol according to the domain of utility of the measured variable.

We divided the material properties according to the logic we adopted, which is the characterization of the material in each state of its life:

- Before elaboration: row material, capacity of the clay to exchange ions (cation exchange capacity) [6]-[8].

- Within the elaboration: principal characteristics affecting the facility given by the material properties to elaborate modified materials. For example, water quantity to obtain malleable (plastic) clay paste (molding for example), liquid limit (Casagrande protocol) and plasticity limits to control the procedures of elaboration according to water percentage present in into the clay sheets.

- After elaboration: measurement of the physical properties (shrinking function of time, mechanical resistance and elasticity modulus, specific surface for ions exchange) after elaboration and the evolution depending on several variables: PEG percentage, time, clay size...

After experimentations, we were able to see the effects of the introducing of the PEG and the clay size on the different properties of the clay material, which presents, for some of the properties, highly significant evolutions. For other properties, no evolution has been observed and we will explain it in the next section.

\section{Results and Discussion}

\subsection{Composition of the Row Material}

\subsubsection{Chemical Composition}

The composition of the row clay was determined by making a triacide attack and the ICP (Inductive Coupled 
Plasma) analysis permitted to determinate the constitution of each sample. Table 3 resumes the composition of the clays according to three essays [9].

Let's see now the major clay crystalline phases present in our material, according to the XRD results.

\subsubsection{XRD Analysis of the Row Material}

The XRD analysis permits us to determine the major crystalline elements present in the clay.

Figure 2 presents the XRD picks observed on the raw material. These picks correspond to the Kaolinite, the Illite (mica), the quartz and calcium carbonate as major and apparent crystalline components.

The results of XRD analysis are giver in Table 4 which presents the mineral present in the natural clay.

After $2 \theta=40^{\circ}$, the majority of the phases correspond to the quartz and calcite (calcium carbonate). So the analysis proves that the clay corresponds to the marl, used as construction material in this region.

Due to the lack of information stored in the data base of the XRD machine, it was difficult to define the crystalline phases detected by the XRD. The machine is able to detect only the quartz and calcite.

This is why we used bibliography for the determination of the clay phases for each corresponding picks, according to two articles mentioned in the reference section ([10] and [11]).

In another process, trying to produce a nanocomposite material between this clay and PEG 6000, it was very difficult to precede to the intercalation of the polymer within the clays sheets. It is due the presence of the great percentage of calcite in the clay.

\subsubsection{Scanning Electronic Microscopy}

We have made some SEM Microscopy to see the structure of the materials (Figure 3).

According to the SEM maximum scale, we cannot see much more microstructure. It is due to historic of the material which has been grinded.

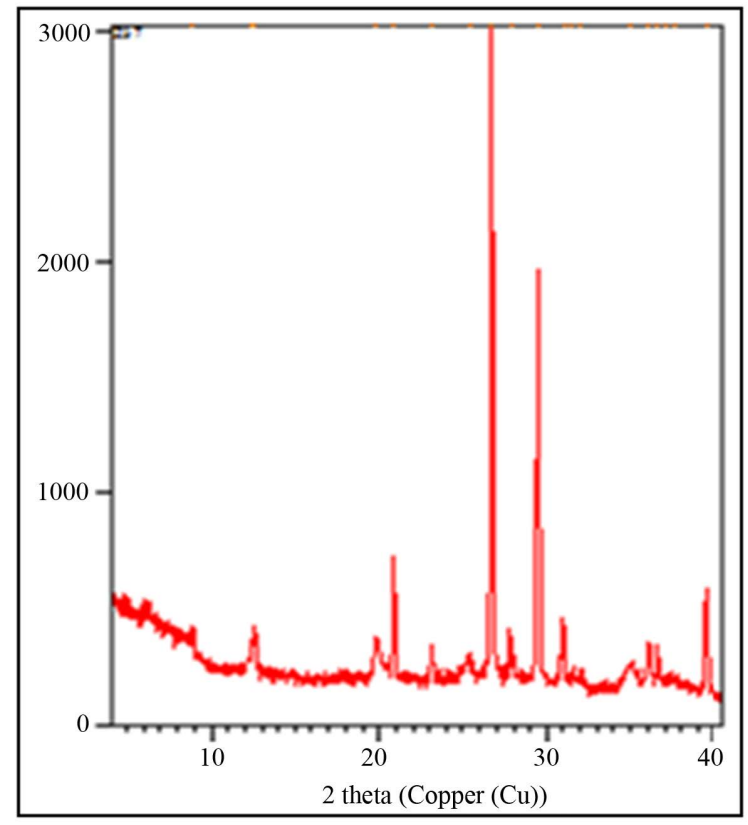

Figure 2. XRD spectrum of row clay materials.

Table 3. Constitution of the clay samples according to [9].

\begin{tabular}{ccccccccccc}
\hline Sampling & $\mathrm{SiO}_{2}$ & $\mathrm{Al}_{2} \mathbf{O}_{3}$ & $\mathbf{C a O}$ & $\mathrm{Fe}_{2} \mathbf{O}_{3}$ & $\mathbf{M g O}$ & $\mathbf{K}_{2} \mathbf{O}$ & $\mathrm{Na}_{2} \mathbf{O}$ & $\mathbf{T i O}_{2}$ & $\mathbf{C r}_{2} \mathbf{O}_{3}$ & $\mathbf{M n O}_{2}$ \\
\hline $\mathbf{1}$ & 44.5 & 14.3 & 7.92 & 5.89 & 3.29 & 1.00 & 0.777 & 0.331 & 1.84 & 0.193 \\
$\mathbf{2}$ & 44.9 & 15.1 & 6.64 & 6.49 & 3.08 & 1.10 & 0.809 & 0.374 & 1.94 & 0.203 \\
$\mathbf{3}$ & 44.5 & 14.8 & 7.20 & 6.20 & 3.18 & 1.01 & 0.832 & 0.359 & 1.95 & 0.207 \\
\hline
\end{tabular}


Table 4. XRD results of the row clay material.

\begin{tabular}{cccc}
\hline Pos. [2 Th.] & Height [cts] & d-spacing [Å] & Mineral \\
\hline 8.7994 & 91.49 & 10.04950 & $\mathrm{M}(001)$ \\
12.3466 & 26.07 & 7.16314 & $\mathrm{~K}(001)$ \\
19.8561 & 168.83 & 4.47149 & $\mathrm{~K}+\mathrm{M}(020)$ \\
20.9208 & 528.89 & 4.24628 & $\mathrm{Q}$ \\
23.1269 & 135.38 & 3.84598 & $\mathrm{Ca}$ \\
25.3796 & 107.06 & 3.50948 & $\mathrm{~K}(002)$ \\
26.6980 & 2882.88 & 3.33909 & $\mathrm{Q}+\mathrm{M}$ \\
29.4753 & 1809.38 & 3.03049 & $\mathrm{Ca}$ \\
31.4364 & 22.32 & 2.84577 & $\mathrm{Ca}$ \\
34.9526 & 112.28 & 2.56713 & $\mathrm{M}$ \\
36.0552 & 213.06 & 2.49111 & $\mathrm{Ca}$ \\
36.5993 & 161.67 & 2.45532 & $\mathrm{M}(131)$ \\
39.5140 & 437.35 & 2.28066 & $\mathrm{Q}+\mathrm{Ca}$ \\
40.3274 & 61.54 & 2.23652 & $\mathrm{Q}$ \\
\hline
\end{tabular}

M: Mica (Illite), K: Kaolinite, Q: Quartz, Ca: Calcite.
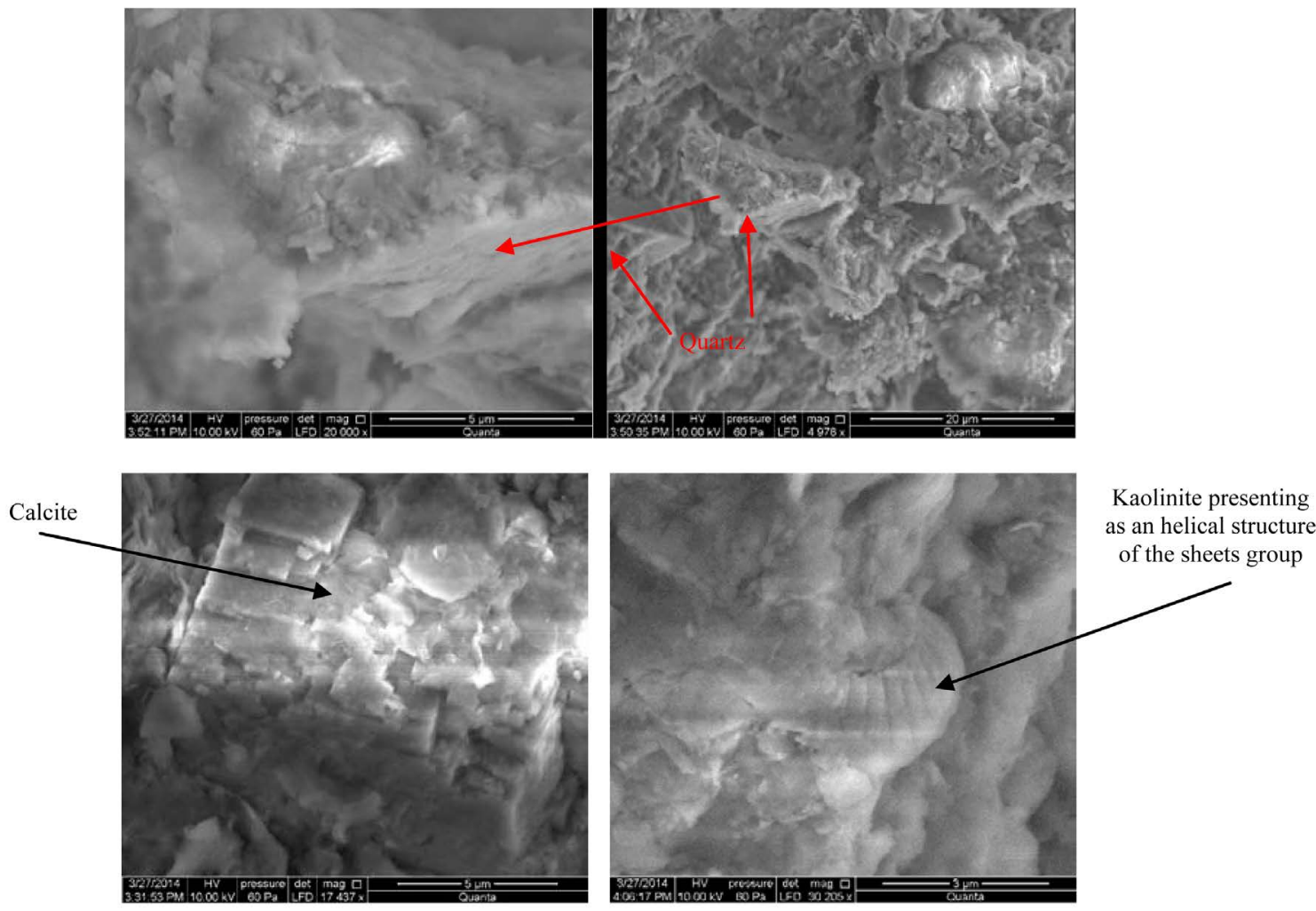

Figure 3. SEM microscopy for the row material. 


\subsection{Physical Properties}

\subsubsection{Atterberg Limits (NF P94-051)}

For each sample, starting from 0 g of PEG 6000 per 6 g of clay to 0.6 g PEG 6000, and according to a step of $0.1 \mathrm{~g}$ of PEG 6000, we measured the liquidity limit by Casagrande protocol and the plasticity limit, according the NF P94-051 standards. Three measurements were used for each sample to have reliable results.

These limits are used in characterization of soil for work earth operations. But in the present study, they permit to understand the mechanical behavior of clay according to the percentage of water. This is an important information for us to estimate the quantity of water to add to the clay in order to elaborate the desirable forms for shrinking and mechanical properties testing.

Figure 4 shows an essay on a manual Casagrande cup of $12 \mathrm{~cm}$ of diameter, for the measurement of liquidity limit.

The corresponding results are presented in Table 5.

We denote in the following discussion the liquidity limit as WL and plasticity limit as WP.

As we introduced in Section 2.1, the two input parameters are the quantity of PEG 6000 mixed in the clay matrix and the clay size (which is fixed on the grinding step).

So to study the influence of the input parameters on the Atterberg limits, we have used the average of each response on each input parameter. We obtain the following results presented in Table 6 and Table 7.

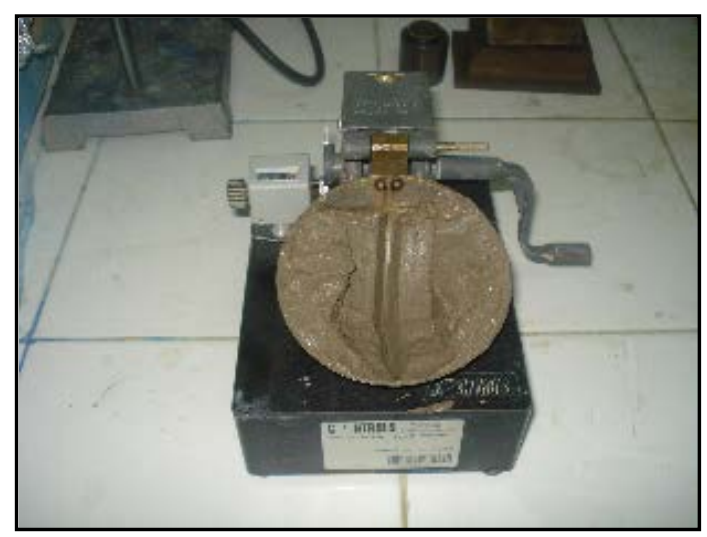

Figure 4. Casagrande essay according to NF P94-051 standards.

Table 5. Atterberg limits and specific surface measurement.

\begin{tabular}{cccccccc}
\hline No. experiment & $\mathbf{W L}$ & $\mathbf{W P}$ & $\mathbf{S S T}\left(\mathbf{m}^{2} / \mathbf{g}\right)$ & No. experiment & WL & WP & SST $\left(\mathbf{m}^{2} / \mathbf{g}\right)$ \\
\hline $\mathbf{1}$ & $27.96 \%$ & $15.58 \%$ & 146.51 & $\mathbf{1 0}$ & $36.17 \%$ & $12.71 \%$ & 146.51 \\
$\mathbf{2}$ & $31.63 \%$ & $14.42 \%$ & 99.42 & $\mathbf{1 1}$ & $35.11 \%$ & $13.28 \%$ & 156.97 \\
$\mathbf{3}$ & $31.97 \%$ & $13.50 \%$ & 115.12 & $\mathbf{1 2}$ & $29.42 \%$ & $12.87 \%$ & 104.65 \\
$\mathbf{4}$ & $28.64 \%$ & $15.42 \%$ & 94.18 & $\mathbf{1 3}$ & $33.92 \%$ & $12.53 \%$ & 151.75 \\
$\mathbf{5}$ & $29.98 \%$ & $14.02 \%$ & 104.65 & $\mathbf{1 4}$ & $33.45 \%$ & $16.31 \%$ & 125.60 \\
$\mathbf{6}$ & $31.22 \%$ & $13.67 \%$ & 120.35 & $\mathbf{1 5}$ & $28.19 \%$ & $12.17 \%$ & 115.12 \\
$\mathbf{7}$ & $36.80 \%$ & $12.61 \%$ & 102.33 & $\mathbf{1 6}$ & $33.05 \%$ & $13.65 \%$ & 120.35 \\
$\mathbf{8}$ & $29.36 \%$ & $12.73 \%$ & 109.90 & $\mathbf{1 7}$ & $33.90 \%$ & $15.38 \%$ & 136.05 \\
$\mathbf{9}$ & $30.13 \%$ & $14.87 \%$ & 104.65 & $\mathbf{1 8}$ & $27.38 \%$ & $12.40 \%$ & 94.19 \\
$\mathbf{1 9}$ & $34.30 \%$ & $21.04 \%$ & 125.28 & $\mathbf{2 1}$ & $34.03 \%$ & $14.31 \%$ & 104.65 \\
$\mathbf{2 0}$ & $34.96 \%$ & $19.26 \%$ & 112.84 & & & & \\
\hline
\end{tabular}


Table 6. WL, WP and SST averages according to PEG weights.

\begin{tabular}{cccc}
\hline PEG 6000 weight (g) per $\mathbf{6}$ g of clay & WL & WP & SST ( $\left.\mathbf{m}^{2} / \mathbf{g}\right)$ \\
\hline 0.00 & $34.43 \%$ & $15.63 \%$ & 115.12 \\
0.10 & $30.52 \%$ & $15.00 \%$ & 120.35 \\
0.20 & $29.95 \%$ & $14.54 \%$ & 106.39 \\
0.30 & $32.10 \%$ & $13.41 \%$ & 107.27 \\
0.40 & $32.79 \%$ & $12.87 \%$ & 125.58 \\
0.50 & $31.05 \%$ & $12.35 \%$ & 130.28 \\
0.60 & $31.44 \%$ & $13.81 \%$ & 116.86 \\
\hline
\end{tabular}

Table 7. WL, WP and SST average according to clay size.

\begin{tabular}{cccc}
\hline Clay size & WL & WP & SST $\left(\mathbf{m}^{2} / \mathbf{g}\right)$ \\
\hline 3 & $32.98 \%$ & $13.85 \%$ & 130.81 \\
5 & $31.97 \%$ & $15.45 \%$ & 115.12 \\
7 & $30.33 \%$ & $13.38 \%$ & 108.39 \\
\hline
\end{tabular}

Figure 5 presents the plot of these results and shows the effect of each of the two parameters, quantity of the PEG added and clay size, on the liquidity limits and Figure 6 corresponds to the effect on the plasticity.

We observe here that the liquidity limit decreases highly by the addition of the PEG 6000 to the natural clay.

\section{Interpretation and Discussion}

- Liquidity Limit:

$\checkmark$ Effect of PEG 6000

In our opinion, and in lower percentage of PEG (from $0.1 \mathrm{~g}$ to $0.3 \mathrm{~g}$ ), the variation is due to mechanical stress effect produced by the molecule of the polymer in a microscopic scale.

The assumption we made is the possibility of an additional swelling given by the PEG 6000 molecules (red arrow of Figure 7(a)), and which could help water to make the clay more liquid. In other words, a lower value of water permits to make the clay more fluid by the addition of PEG 6000 which increase the distance between clay sheets groups and by the way affects the compactness and rigidity (in microscopic scale) of the clay.

For higher value of PEG 6000 (0.4 g to $0.6 \mathrm{~g}$ ), we make the assumptions that at a certain limit higher value ( $0.4 \mathrm{~g}$ in this case), the molecules of the polymer begin to form some groups with the clay sheets, taking into account that no intercalation has been observed. So these groups will be more rigid and we will have to add more water to move from plastic state to liquid state.

Normally, in lower value of the polymer, water will attack instantly the clay by going through the porosity and the clay inter-sheet spaces and will cause the destruction of the links between clay sheets. But by the addition of non neglected masses of PEG 6000, water will have two roles; the first is the increasing of the intersheets distance. The second will be the destruction of PEG 6000 links to attack the clay grouped and reinforced by the polymer.

In Figure 7, we try to schematize these assumptions.

\section{$\checkmark$ Effect of the Clay Size}

From Figure 5(b), it is clear that the clay size has an important effect on the liquidity limit. For this study, the scale of the clay size can be read from the greater size (3) to the finest one (7).

Plasticity evaluates by increasing from the finest size to the greater one. In other words, for higher sizes, the material needs more water to move from the solid state to past state. It is due to the greater spaces existing around clay groups (grains) which have to be filled by water if the size of the grains increases.

\section{- Plasticity Limit:}

\section{$\checkmark$ Effect of PEG 6000}

From Figure 6(a), it is clear that the plasticity limit decreases when adding the PEG 6000 to the natural clay. 


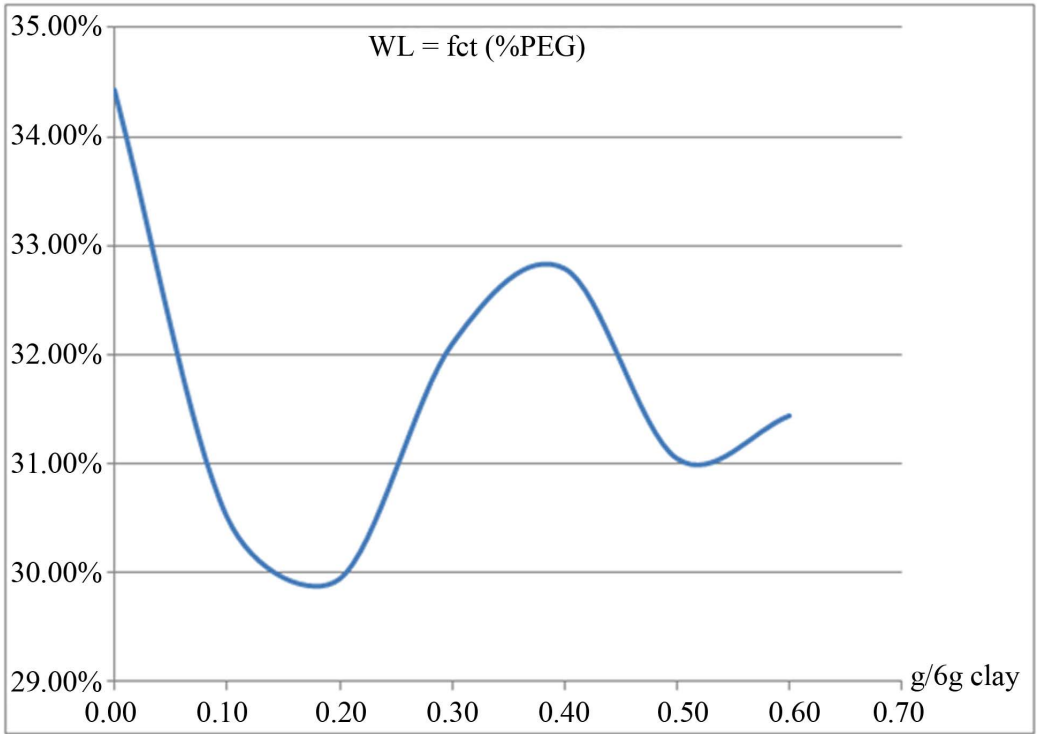

(a)

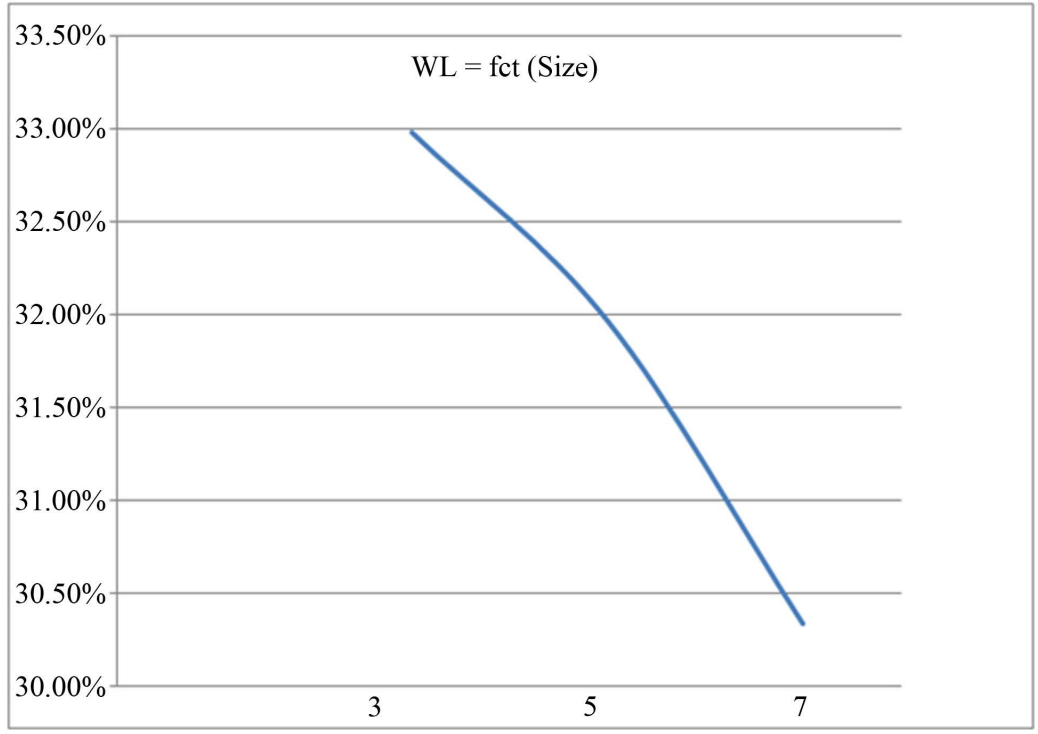

(b)

Figure 5. Liquidity limit according to PEG quantity (g/6g of clay) (a) and clay size (b).

Our interpretation is that the clay does not need more water to move from solid state to past state because of the presence of the polymer.

PEG 6000 helps so to obtain better malleability (past state) by decreasing the percentage of water. From a microscopic view, PEG 6000 increases the coherence of clay sheets groups by linking between them.

It could be explained by the creation of some PEG 6000 cations due to its dissolution in water/clay solution, and by the way the link factor will be the cationic exchange capacity of the clay [3]; or only by mechanical effect, where the PEG 6000 gives more rigidity to the material at lower water percentage.

\section{$\checkmark$ Effect of Clay Size}

We can see on Figure 6(b) that the plasticity (percentage of water to move from solid state to past state) attains its maximum on the value 5 . But we cannot make any suggestion at this stage. We have no tend and the number of data, even if it exceeds 20 essays, we think that it will be more scientific to enlarge the interval of clay size to have more information and, by this way, extract a real conclusion according to the clay size effect. 


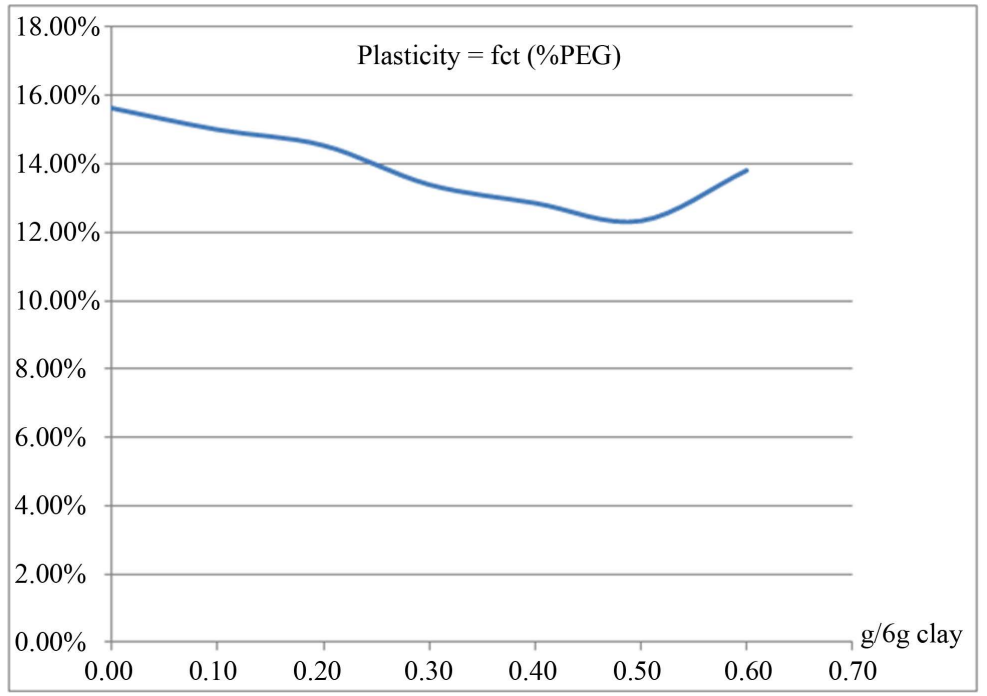

(a)

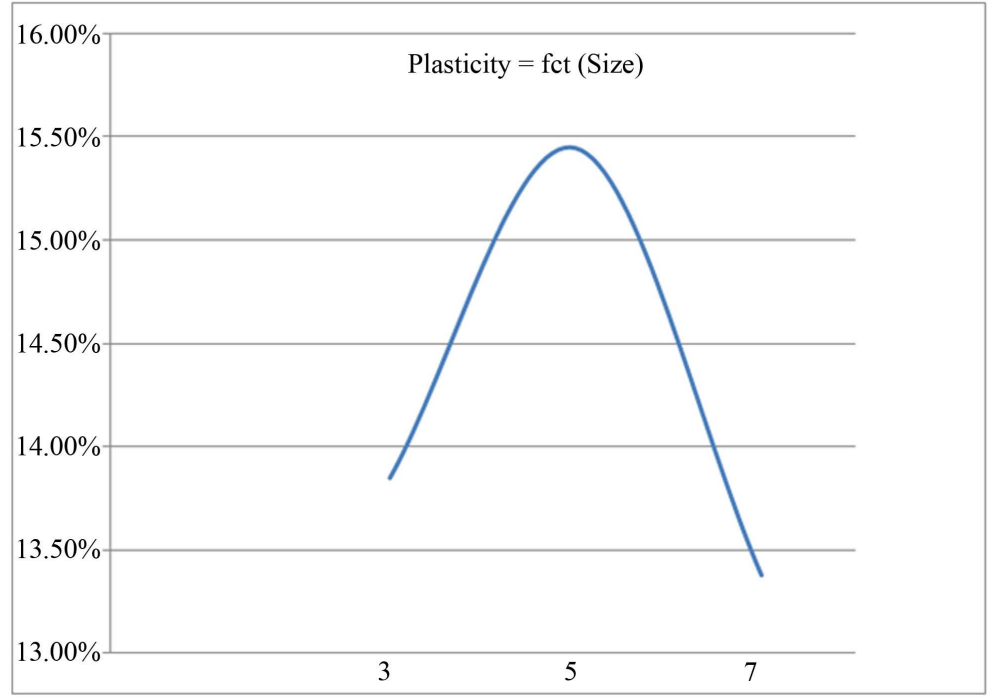

(b)

Figure 6. Plasticity limit according to PEG quantity (g/6g of clay) (a) and clay size (b).

3.2.2. Specific Surface (Cationic Exchange) and Methylen Blue Essays (XP P 18-540)

The Methylen Blue (MB) essay is a simple essay which permits to estimate approximately the capacity of adsorbing, specific surface or cation exchange capacity, of a fine size clay $(80 \mu \mathrm{m})([6]$ and [7]).

In clay, two different phenomena could occur: mechanical intercalation between the nanoclay sheets or electrical effect defined by cationic exchange of clay [12].

\section{Remark}

In our case, we have make simple grinding of the two substances, and XRD analysis and comparison between the new and the old material does not give any difference between them. The picks are fixed in their position, so no intercalation has occurred.

But it was necessary to make MB characterization for future works when we will adopt humid way to elaborate the nanocomposite.

Table 5 presents the detail of the specific surface $\left(\mathrm{m}^{2} / \mathrm{g}\right)$ according to the volume of MB adsorbed of all the materials. The specific surface is denoted SSA and it is calculated by the Equation (1) [13]. 


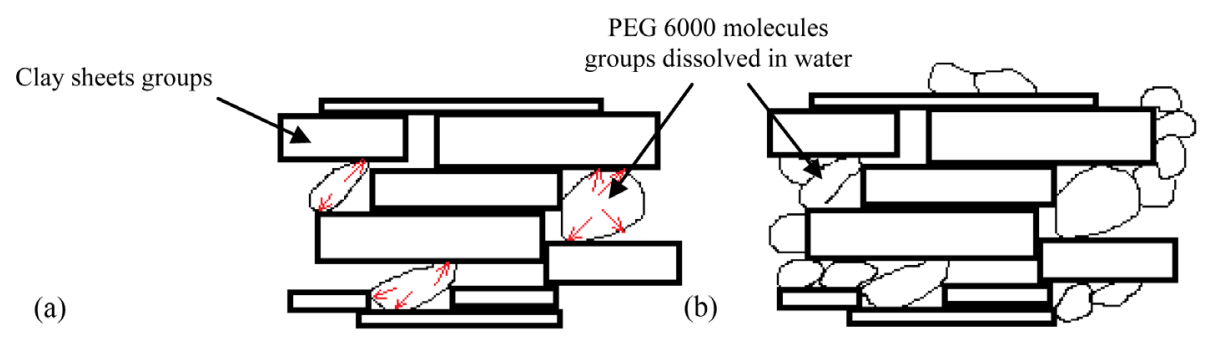

Figure 7. Effect of PEG 6000 on the mechanical behavior of the composite before water addition. (a) At low values ( $0.1 \mathrm{~g}$ to $0.4 \mathrm{~g}$ ), additional stress modeled by red arrow helps the effect of water on the dispersion of clay sheets; (b) At higher values ( $0.5 \mathrm{~g}$ and $0.6 \mathrm{~g}$ ) water has to dissolve the PEG groups firstly to access to clay groups.

$$
S S A=20.93 \frac{V B}{m}
$$

where $V B$ is the volume of methylene blue; $m$ is the mass of clay; 20.93 is a constant depending on the adsorbed substance. In our case it is the methylen blue.

\section{Interpretation and Assumptions}

$\checkmark$ Values of Specific Surface

The values of specific surface SST encounter here correspond to the values of specific surface of the illite, a kind of Micas, which vary from $100 \mathrm{~m}^{2} / \mathrm{g}$ to $175 \mathrm{~m}^{2} / \mathrm{g}$.

\section{$\checkmark$ Effect of PEG 6000}

We can see that the effect of PEG 6000 is not too significant. The values oscillate around an average of $117.40 \mathrm{~m}^{2} / \mathrm{g}$.

The curve does not present any trend. Hence, we deduce that the PEG 6000 addition with a grinding/mixing method can't modify the specific surface. In other word the cationic exchange capacity is not modified due to the macroscopic aspect of the elaboration method, grinding. But we will see that the clay size value is very significant.

\section{$\checkmark$ Effect of Clay Size}

Figure 8(b) shows that the clay size affects greatly the adsorbing capacity of the clay. The specific surface decreases with the clay size diminution.

This decreasing can be understood by the destruction of the clay sheets which are electrically charged, positively or negatively (capacity of ions exchange). So when broking a sheet, the capacity of attracting other cations or anions will be reduced, and we cannot say that the sum of the capacity exchange of a set of little sheets created by the broking correspond to the whole capacity exchange of the initial clay sheet.

Christidis [14] has modeled this description with an easy scheme presented on Figure 9.

After destruction, Figure 9(c), the only party which can significantly affect the ionic exchange is the crystalline phase. The amorphous phase, which is in the origin composed by the same clay layer sheets, has low reactivity and will not contribute to the ionic exchange of the resulting material.

On Figure 10, the TEM microscopy permits us to see clearly the constitution of an amorphous phase, which presents several clay sheets dispersed in space.

The results we obtain confirm this thesis by the curve reported on Figure 8(b).

The greater is the clay size, the higher is the specific surface. It evaluates/decreases to the lowest value according to the little value of clay size, having approximately a linear behavior (Figure 7(b)).

\subsubsection{Shrinking, Function of Time, Polymer Percentage and Clay Size}

On the stage of construction material processing, the shrinking is considered as the major effect that will has to be taken into account.

The shrinking corresponds to the reduction of the volume of the piece (bricks or cylinders), which is caused by the evaporation of residual water that exists after the elaboration.

The variation of the volume can be understood like the diminution of at least one dimension of the piece. That is why, linked to the notion of rigidity of the material, the variation of the dimensions causes internal constraints 


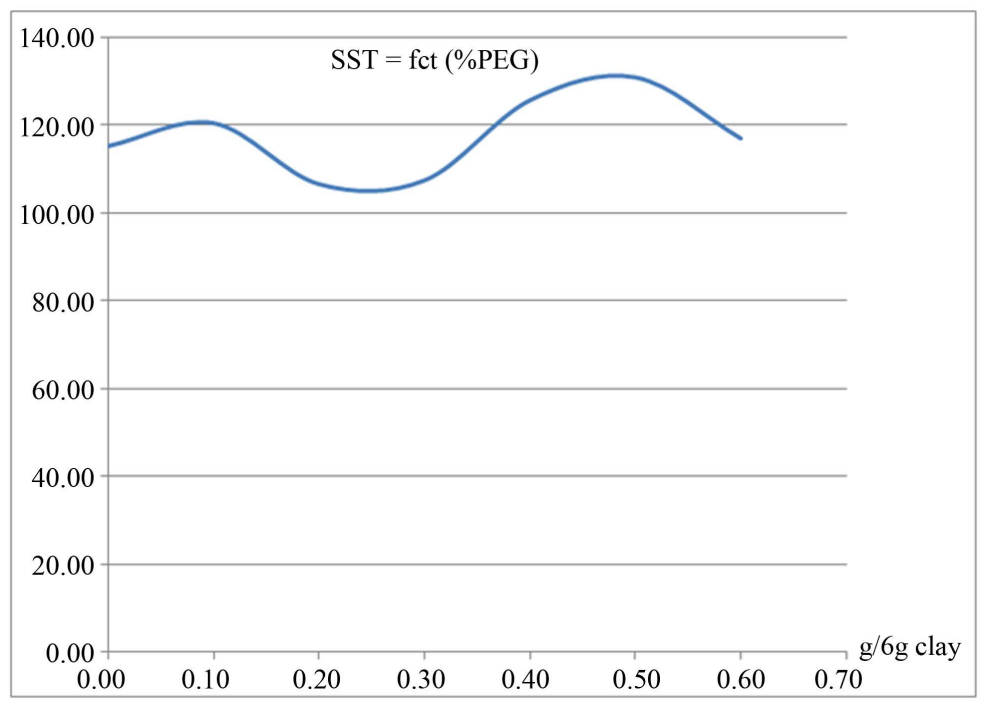

(a)

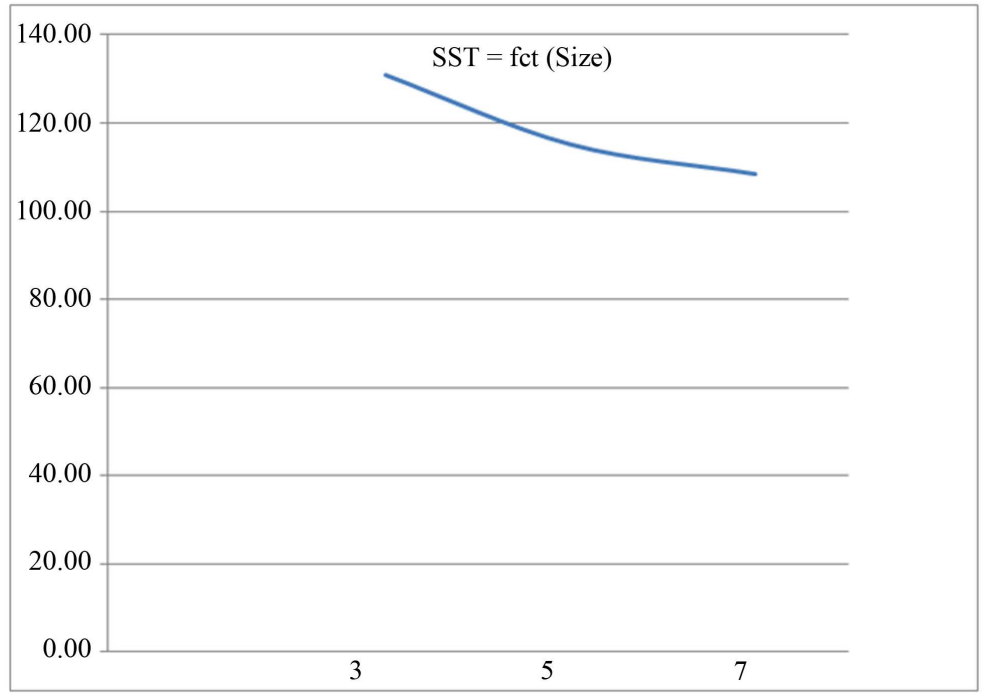

(b)

Figure 8. Specific surface according to PEG quantity (g/6g of clay) (a) and clay size (b).

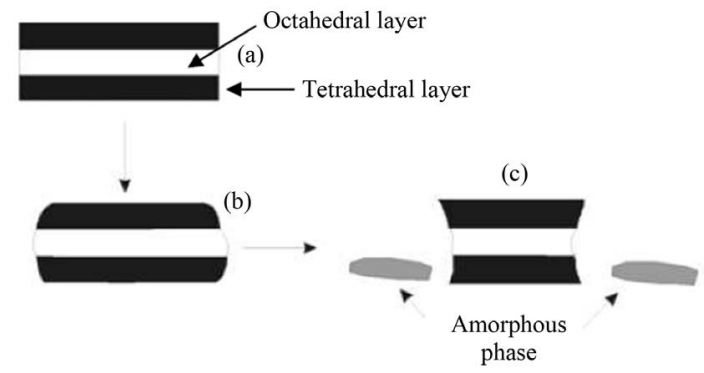

Figure 9. Description of the different steps of the destruction of a clay (taken from [15]). (a) Schematic representation of the clay; (b) Grinding beginning: the tetrahedral layer, the tetrahedral layers are destructed the first; (c) Destruction of the octahedral layer forming an amorphous layer. 


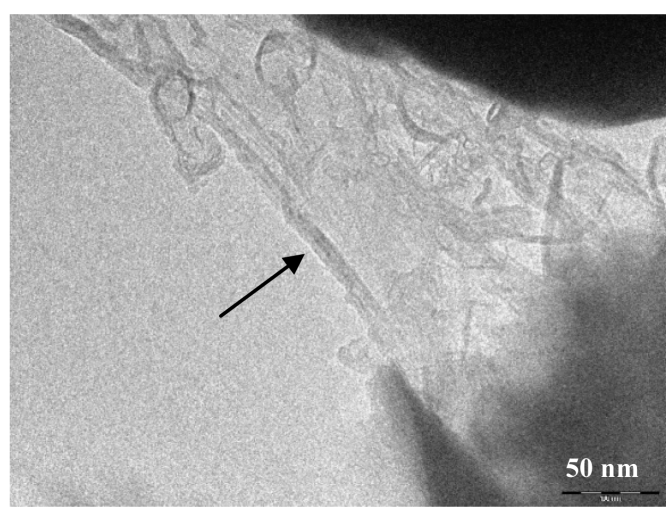

(a)

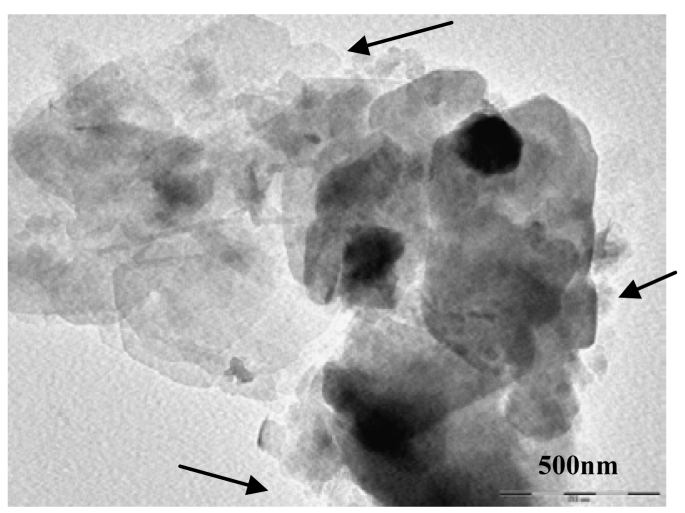

(b)

Figure 10. (a) TEM microscopy and amorphous phase caused by the grinding method (0.5 g PEG 6000/6g clay); (b) Amorphous phases (dispersion of little clay sheets) shown by the black arrows (0.6 g PEG 6000/6g clay).

which, if exceeding the mechanical resistance of the material, causes the cracking of a part or the entire piece.

That is why, on concrete construction, it is important to make the concrete humid, especially on the first days after concrete pouring [16], to maintain the quantity of water constant and the volume of concrete also, when it is on the stage of consolidation. By the way the consolidation of the material will be on good conditions, when there are no internal constraints caused by an excess of shrinking.

In this section, we will show how the PEG 6000 can limit the shrinking even in the case of simple grinding.

In a future works, we will show the great effect of the elaboration method of the row material on this parameter. Shrinking was reduced to a minimal value by adding PEG 6000 with humid method.

Figure 11 shows some examples of cylinders we elaborated for the measurement of shrinking and mass diminution.

\section{1) Shrinking average according to PEG 6000}

Three measurements of the diameter and length were adopted each day for height days; and the average was calculated for the results plotting.

Table 8 presents the results of the average of reduction of the diameter and length and also the mass reduction according to PEG 6000 values.

The plotting of the evolution of shrinking according to PEG 6000 mass added is presented on Figure 12.

We observe high variation of the shrinking especially on the first four days after cylinder elaboration. After that, the shrinking evolution curve takes an asymptotic behavior due to the stabilization of the shrinking phenomena.

Cement presents the samfe shrinking behavior. Eurocode 2 standards, Section 1.1, present the equation of shrinking ratio as (see Figure 13):

$$
r(t)=1-e^{-\tau t}
$$

$\tau$ is a characteristic constant which depends on the material. This constant is calculated according to the Equation (3) (tangent line at the point $t=0$ ).

$$
\tau=r^{\prime}(0)
$$

According to the curves of Figure 14, the shrinking for all parameters, diameter, length and the mass follow the same look as on the Figure 13.

So we can see that the deformations/shrinking denoted in our case correspond to the same look as the shrinking function of cement, because of that the clay in on the basis of cement processing. It could be possible to model the $r(t)$ function for all the samples, but for a first time, in this article, we will show the effect of the input which are PEG 6000 doping quantity and cay size.

\section{2) Shrinking average according to clay size}

Table 9 presents the averages of shrinking according to the clay size. The corresponding results are plotted on Figure 14, to see the influence of the clay size on shrinking. 


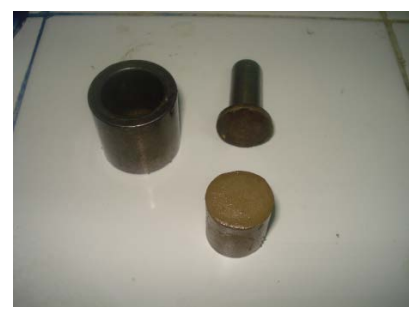

(a)

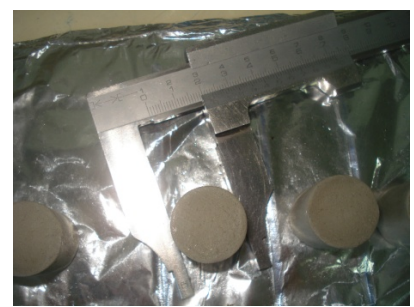

(b)

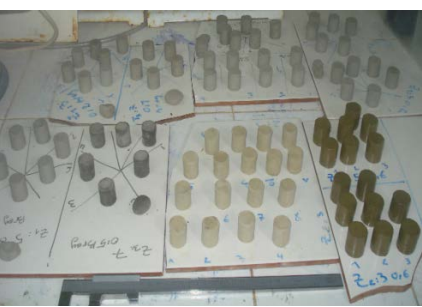

(c)

Figure 11. Cylinder samples elaborated for shrinking measurement. We adopted at least four cylinders by essay, according to our experiments plan.

Table 8. Average of samples shrinking according to PEG 6000 percentage.

\begin{tabular}{|c|c|c|c|c|c|c|c|c|c|}
\hline \multirow{2}{*}{$\begin{array}{l}\text { PEG Qty } \\
\text { (g)/6g clay }\end{array}$} & \multirow{2}{*}{ Day } & \multicolumn{3}{|c|}{ Shrinking according to PEG 6000} & \multirow{2}{*}{$\begin{array}{c}\text { PEG Qty (g)/6 } \\
\text { g clay }\end{array}$} & \multirow{2}{*}{ Day } & \multicolumn{3}{|c|}{ Shrinking according to PEG 6000} \\
\hline & & Diameter & Length & Mass loss & & & Diameter & Length & Mass loss \\
\hline \multirow{8}{*}{0.0} & 1 & & & & \multirow{8}{*}{0.4} & 1 & & & \\
\hline & 2 & $3.56 \%$ & $4.26 \%$ & $7.17 \%$ & & 2 & $2.33 \%$ & $2.71 \%$ & $5.16 \%$ \\
\hline & 3 & $7.19 \%$ & $6.94 \%$ & $11.62 \%$ & & 3 & $4.94 \%$ & $5.47 \%$ & $9.91 \%$ \\
\hline & 4 & $9.07 \%$ & $8.79 \%$ & $14.60 \%$ & & 4 & $6.21 \%$ & $6.43 \%$ & $11.63 \%$ \\
\hline & 5 & $9.80 \%$ & $9.50 \%$ & $21.86 \%$ & & 5 & $6.86 \%$ & $7.05 \%$ & $12.89 \%$ \\
\hline & 6 & $10.06 \%$ & $9.74 \%$ & $20.33 \%$ & & 6 & $7.57 \%$ & $7.62 \%$ & $13.89 \%$ \\
\hline & 7 & $10.00 \%$ & $9.83 \%$ & $21.23 \%$ & & 7 & $7.62 \%$ & $8.13 \%$ & $14.84 \%$ \\
\hline & 8 & $9.85 \%$ & $9.94 \%$ & $21.66 \%$ & & 8 & $8.33 \%$ & $8.84 \%$ & $15.45 \%$ \\
\hline \multirow{8}{*}{0.1} & 1 & & & & \multirow{8}{*}{0.5} & 1 & & & \\
\hline & 2 & $3.23 \%$ & $3.67 \%$ & $5.01 \%$ & & 2 & $2.53 \%$ & $2.46 \%$ & $5.10 \%$ \\
\hline & 3 & $5.93 \%$ & $6.12 \%$ & $8.68 \%$ & & 3 & $5.10 \%$ & $5.35 \%$ & $9.44 \%$ \\
\hline & 4 & $7.93 \%$ & $8.19 \%$ & $12.61 \%$ & & 4 & $6.48 \%$ & $6.41 \%$ & $11.71 \%$ \\
\hline & 5 & $8.54 \%$ & $9.21 \%$ & $16.99 \%$ & & 5 & $7.31 \%$ & $6.99 \%$ & $12.76 \%$ \\
\hline & 6 & $8.92 \%$ & $9.27 \%$ & $19.47 \%$ & & 6 & $8.11 \%$ & $7.76 \%$ & $13.97 \%$ \\
\hline & 7 & $8.67 \%$ & $9.57 \%$ & $20.38 \%$ & & 7 & $8.45 \%$ & $8.42 \%$ & $15.24 \%$ \\
\hline & 8 & $8.67 \%$ & $9.47 \%$ & $21.20 \%$ & & 8 & $9.01 \%$ & $7.63 \%$ & $15.06 \%$ \\
\hline \multirow{8}{*}{0.2} & 1 & & & & \multirow{8}{*}{0.6} & 1 & & & \\
\hline & 2 & $2.61 \%$ & $3.35 \%$ & $5.55 \%$ & & 2 & $1.00 \%$ & $1.60 \%$ & $4.02 \%$ \\
\hline & 3 & $3.95 \%$ & $5.76 \%$ & $10.22 \%$ & & 3 & $3.75 \%$ & $3.74 \%$ & $6.74 \%$ \\
\hline & 4 & $6.74 \%$ & $8.56 \%$ & $14.96 \%$ & & 4 & $4.66 \%$ & $4.64 \%$ & $8.46 \%$ \\
\hline & 5 & $8.45 \%$ & $9.33 \%$ & $18.83 \%$ & & 5 & $5.65 \%$ & $5.59 \%$ & $9.66 \%$ \\
\hline & 6 & $8.41 \%$ & $9.29 \%$ & $19.72 \%$ & & 6 & $5.84 \%$ & $6.03 \%$ & $10.82 \%$ \\
\hline & 7 & $8.45 \%$ & $9.46 \%$ & $20.40 \%$ & & 7 & $6.59 \%$ & $6.75 \%$ & $11.96 \%$ \\
\hline & 8 & $8.43 \%$ & $9.56 \%$ & $21.07 \%$ & & 8 & $7.09 \%$ & $7.18 \%$ & $12.59 \%$ \\
\hline \multirow{8}{*}{0.3} & 1 & & & & & & & & \\
\hline & 2 & $2.89 \%$ & $2.76 \%$ & $4.30 \%$ & & & & & \\
\hline & 3 & $4.20 \%$ & $4.05 \%$ & $7.46 \%$ & & & & & \\
\hline & 4 & $5.61 \%$ & $5.48 \%$ & $11.14 \%$ & & & & & \\
\hline & 5 & $7.80 \%$ & $7.20 \%$ & $14.38 \%$ & & & & & \\
\hline & 6 & $8.10 \%$ & $7.75 \%$ & $16.11 \%$ & & & & & \\
\hline & 7 & $8.41 \%$ & $8.24 \%$ & $16.77 \%$ & & & & & \\
\hline & 8 & $8.29 \%$ & $8.30 \%$ & $18.16 \%$ & & & & & \\
\hline
\end{tabular}




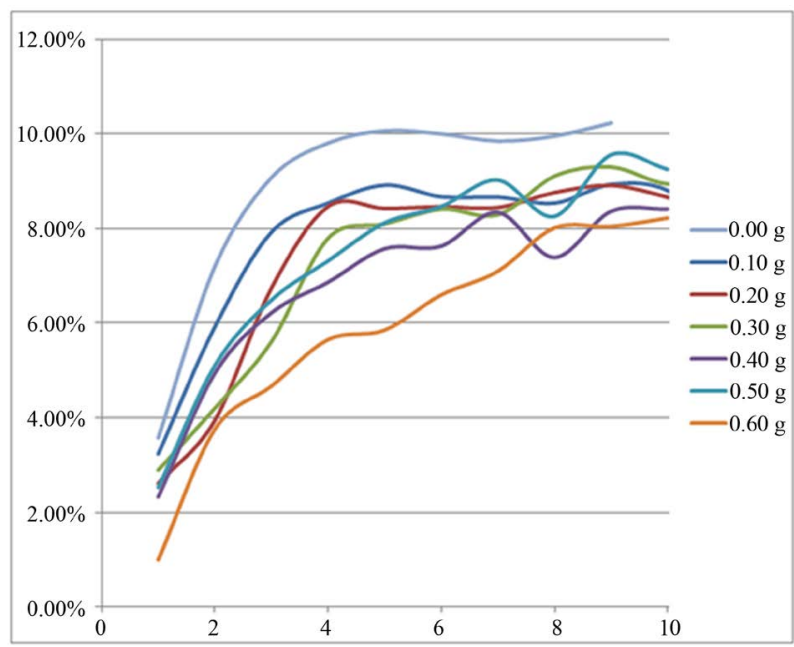

(a)

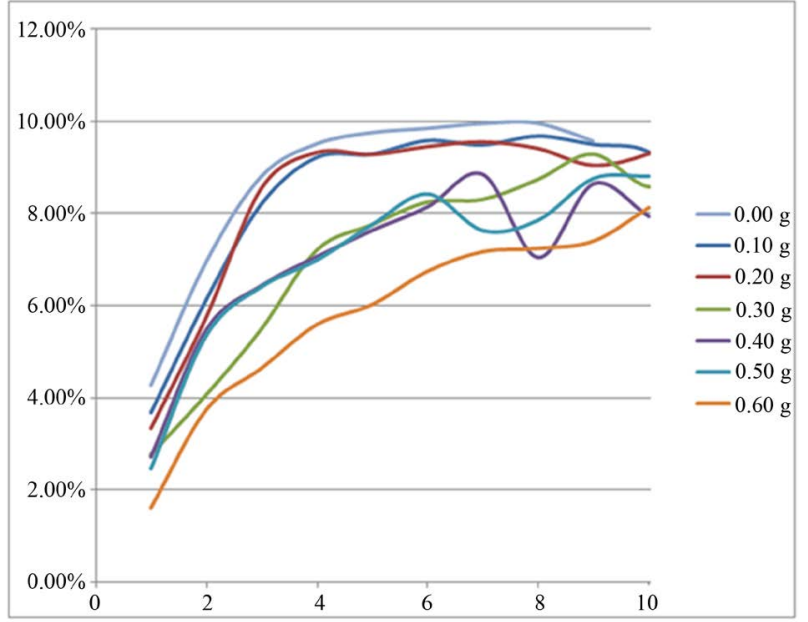

(b)

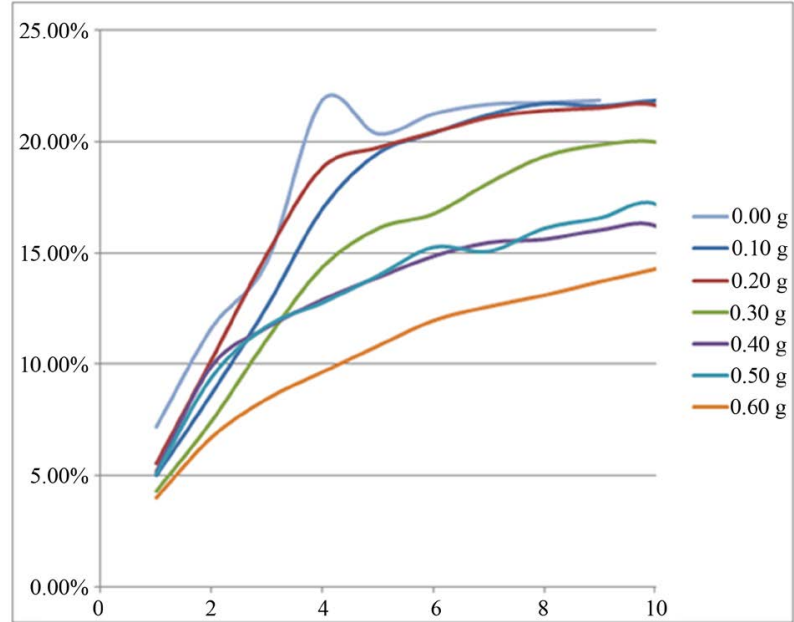

(c)

Figure 12. Shrinking/diminution average of the cylinder according to PEG 6000 doping. (a) Diminution of the diameter; (b) Diminution of the length; (c) Mass diminution. 


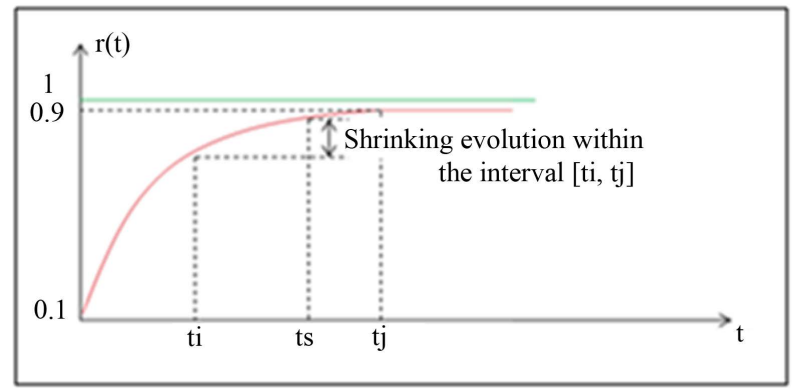

Figure 13. Shrinking function evolution of cement according to Eurocode 2, Section 1.1.

Table 9. Average of samples shrinking according to PEG 6000 percentage.

\begin{tabular}{cccccccccccccc}
\hline & \multicolumn{3}{c}{ Shrinking according to clay size } & \multicolumn{4}{c}{ Shrinking according to clay size } & \multicolumn{3}{c}{ Shrinking according to clay size } \\
\hline Size & Day & Diameter & Length & Mass & Size Day & Diameter & Length & Mass & Size & Day & Diameter & Length & Mass \\
\hline 1 & & & & 1 & & & & & 1 & & & \\
2 & $2.93 \%$ & $3.54 \%$ & $5.64 \%$ & 2 & $3.52 \%$ & $3.65 \%$ & $5.34 \%$ & 2 & $2.28 \%$ & $2.55 \%$ & $4.59 \%$ \\
3 & $5.34 \%$ & $5.42 \%$ & $10.34 \%$ & 3 & $5.20 \%$ & $5.22 \%$ & $9.02 \%$ & 3 & $4.40 \%$ & $4.88 \%$ & $8.24 \%$ \\
3 & 4 & $7.22 \%$ & $7.25 \%$ & $13.41 \%$ & 4 & $7.07 \%$ & $6.78 \%$ & $12.78 \%$ & 4 & $6.12 \%$ & $6.53 \%$ & $11.17 \%$ \\
5 & $8.18 \%$ & $8.13 \%$ & $16.29 \%$ & 5 & $8.47 \%$ & $8.20 \%$ & $16.32 \%$ & 5 & $7.43 \%$ & $7.73 \%$ & $15.95 \%$ \\
6 & $8.68 \%$ & $8.36 \%$ & $17.90 \%$ & 6 & $8.74 \%$ & $8.56 \%$ & $18.38 \%$ & 6 & $7.76 \%$ & $8.29 \%$ & $15.89 \%$ \\
7 & $8.74 \%$ & $8.86 \%$ & $18.30 \%$ & 7 & $8.81 \%$ & $8.88 \%$ & $19.33 \%$ & 7 & $7.99 \%$ & $8.55 \%$ & $16.92 \%$ \\
8 & $9.24 \%$ & $9.45 \%$ & $21.32 \%$ & 8 & $8.83 \%$ & $8.77 \%$ & $20.25 \%$ & 8 & $8.22 \%$ & $8.72 \%$ & $17.46 \%$ \\
\hline
\end{tabular}

Indeed, we observe that shrinking is decreasing from the highest size 3 to the lowest size 7.

Interpretation and Discussion

\section{Effect of PEG 6000}

As we can see on the curves, the row material, with $0 \%$ of PEG, have the maximum of shrinking and it decreases by adding more and more PEG 6000.

It is clear that the PEG 6000 has a significant effect on the variation of the clay shrinking.

We can explain this effect by the fact that the PEG 6000 fills the spaces or sites that exist into the clay, and after water evaporation (water is used to elaborate clay past for cylinder making), the polymer stops, by mechanical effect, the movement of clay sheets.

It will reduce so on the internal constraints due to shrinking and avoid clay cylinder cracking.

\section{$\checkmark$ Effect of the Clay Size}

According to the results of Table 9 and Figure 14, clay size has also an important effect on the shrinking diminution.

As we saw in Section 3.2-b), when the clay size is low, it gives more amorphous phases which decrease the ionic exchange within the clay.

We assume so that in the processing step of the cylinders, for low clay size, water adsorbing was low from the beginning, so symmetrically the corresponding shrinking was so.

\section{Conclusions}

In this paper we have tried to valorize natural clay by adding PEG 6000. We have adopted a complete experimentation plan according to 6 values of PEG 6000 (Polyethylene Glycol 6000) quantity and three clay size values.

Even if we used simple grinding (dried method), we have obtained important results regarding physical/geo- 


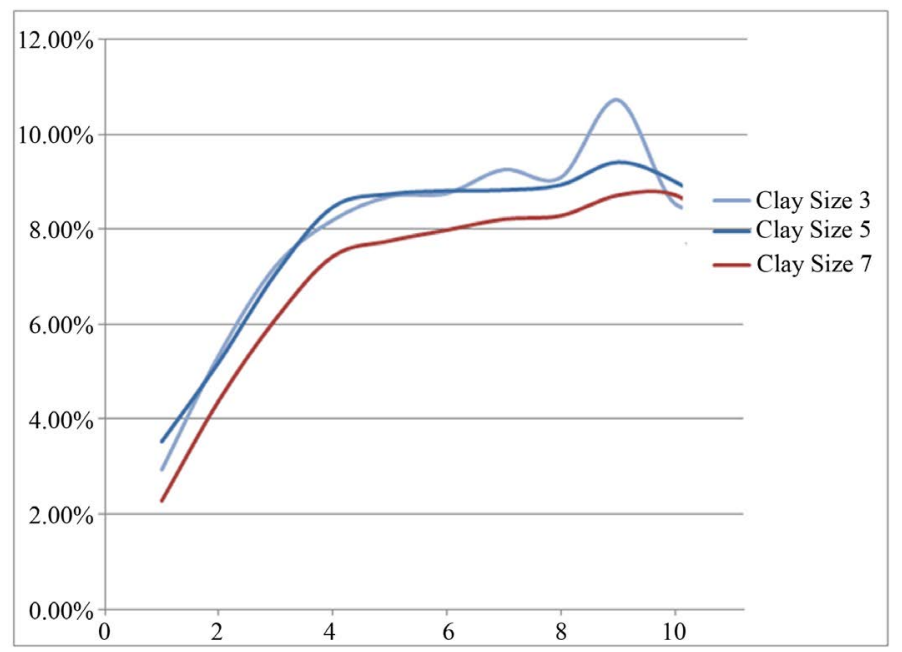

(a)

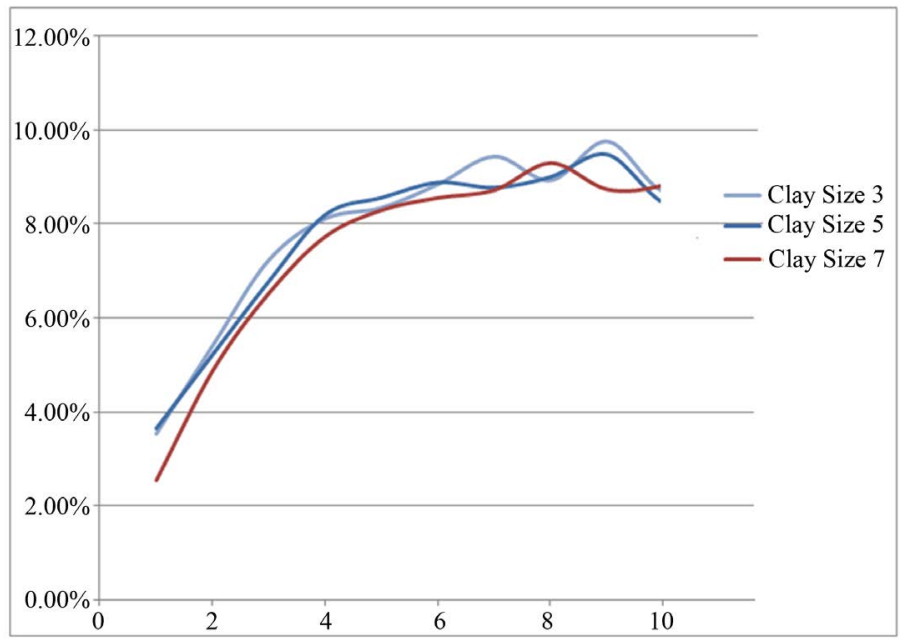

(b)

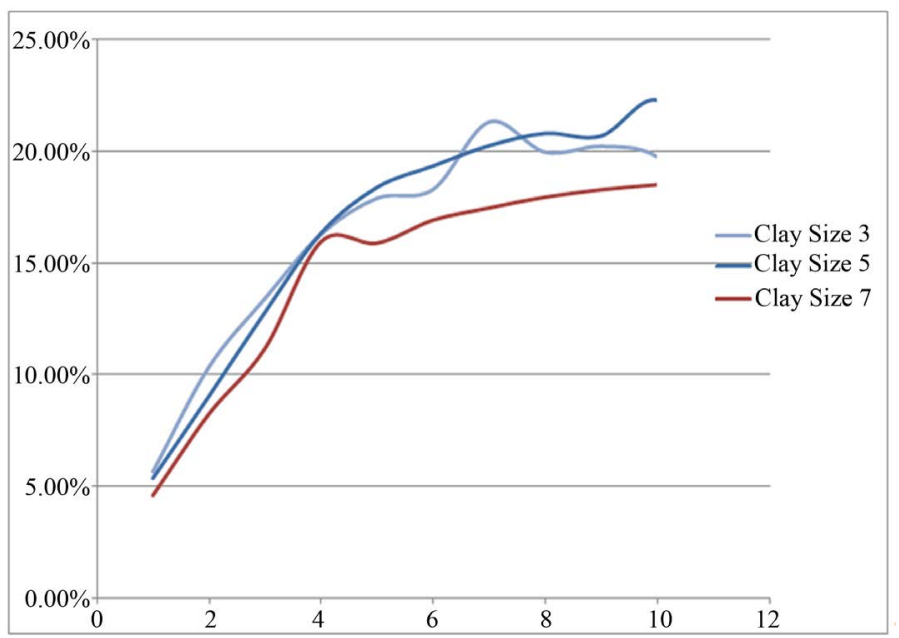

(c)

Figure 14. Shrinking/diminution average of the cylinder according to clay size. (a) Diminution of the diameter; (b) Diminution of the length; (c) Mass diminution. 
technical properties:

$\checkmark$ The modification of the liquidity and plasticity limits (Figure 5 and Figure 6) according to PEG 6000 percentage and clay sizes;

$\checkmark$ The modification of the specific surface (Figure 8), which will permit us to estimate the effect on the swelling and by the way the effect on the material shrinking (Figure 12 and Figure 14), according on the two input parameters.

Thus, we propose so to integrate these results in construction and geotechnical field to look for some application as ground reinforcement [16] and classical material processing.

\section{Acknowledgements}

A special thank to "UATRS (Unités d'Appui Technique à la Recherche Scientifique)" of the CNRST (Centre Nationale de Recherche Scientifique et Technique), Rabat-Morocco, to permit us to make TEM microscopy and also for "Cité de l'Innovation”, the research center of "Sidi Mohamed Ben Abdellah University”, for the help on SEM microscopy.

Thank you for Mr. AMRANI, from Biology department, who gave us the necessary quantity of PEG 6000 for the essays.

\section{References}

[1] Szabo, T., et al. (2007) Magnetic Iron Oxide/Clay Composites: Effect of the Layer Silicate Support on the Microstructure and Phase Formation of Magnetic Nanoparticles. Nanotechnology, 18, 285602. http://dx.doi.org/10.1088/0957-4484/18/28/285602

[2] Fatiha, A. (2008) Elaboration et caractérisation de Nanocomposites Cellulose Montmorillonite. Magister Report, Engineering Science Faculty, Boumerdes University, Boumerdes.

[3] Burba $3^{\text {rd }}$, J.L. (1981) Adsorption of Ethylene Glycol on Amine-Substituted Montmorillonites. Clays and Clay Minerals, 29, 60-66. http://dx.doi.org/10.1346/CCMN.1981.0290109

[4] Theng, B.K.G. (1982) Clay-Polymer Interactions: Summary and Perspectives. Clays and Clay Minerals, 30, 1-10. http://dx.doi.org/10.1346/CCMN.1982.0300101

[5] Benchabane, A. and Bekkour, K. (2004) Etude de l'effet d'un polymère anionique su le comportement rhéologique de suspensions de bentonite. 39ème Colloque annuel du Groupe Français de Rhéologie, Mulhouse.

[6] Yukselen, Y. and Kaya, A. (2008) Suitability of the Methylene Blue Test for Surface Area, Cation Exchange Capacity and Swell Potential Determination of Clayey Soils. Engineering Geology, 102, 38-45. http://dx.doi.org/10.1016/j.enggeo.2008.07.002

[7] Kaewprasit, C., et al. (1998) Quality Measurement, Application of Methylene Blue Adsorption to Cotton Fiber Specific Surface Area Measurement: Part I. Methodology. The Journal of Cotton Science, 2, 164-173.

[8] Hang, P.T. and Brindley, G.W. (1970) Methylene Blue Absorption by Clay Minerals. Determination of Surface Areas and Cation Exchange Capacities (Clay-Organic Studies XVIII). Clays and Clay Minerals, 18, 203-212. http://dx.doi.org/10.1346/CCMN.1970.0180404

[9] Mesrar, L. (2013) Caractérisation géotechnique minéralogique technologique des marnes miocènes du couloir sud riffan (Taza-Fès): Etude et valorisation. Sciences and Techniques Ph.D. Dissertation, Sidi Mohamed Ben Abdellah University, Morocco.

[10] Lalmi, K. (2008) Etude par Diffraction des RX de matériaux à base de kaolin de KT2 et DD. Magister Report, SCIENCES Faculty, Mentouri-Constantine University.

[11] Rebiai, L., et al. (2010) Caractérisation de la Kaolinite et du Mica dans un kaolin enrichi. Sciences \& Technologie A, 31, 20-25.

[12] Pavlidou, S. and Papaspyrides, C.D. (2008) A Review on Polymer-Layered Silicate Nanocomposites. Progress in Polymer Science, 33, 1119-1198. http://dx.doi.org/10.1016/j.progpolymsci.2008.07.008

[13] Santamarina, J.C., Klein, Y.H. and Prencke, E. (2002) Specific Surface: Determination and Relevance. Canadian Geotechnical Journal, 39, 233-241. http://dx.doi.org/10.1139/t01-077

[14] Christidis, G.E., Dellisanti, I., Valdre, G. and Makri, P. (2005) Structural Modifications of Smectites Mechanically Deformed under Controlled Conditions. Clay Minerals, 40, 511-522. http://dx.doi.org/10.1180/0009855054040188

[15] Gautier, M. (2008) Interactions entre argile ammoniée et molécules organiques dans le contexte du stockage des déchets. Cas de molécules à courtes chaînes. Ph.D. Dissertation, Orléans University, Orléans.

[16] Lefebvre, D. (2002) Effets du retrait-fluage sur les structures en béton précontraint. 4e Conférence spécialisée en génie des structures de la Société canadienne de génie civil, Montréal, 5-8 June 2002. 